\title{
DE NUEVO SOBRE LAS COMPETENCIAS DE NAVARRA EN LA LEY ORGANICA DE REINTE- GRACION Y AMEJORAMIENTO DEL REGIMEN FORAL DE NAVARRA (*)
}

340.148 .2 (46. Navarra)

por

\section{Manuel Pulido Quecedo}

Letrado del Parlamento de Navarra. Profesor Asociado de Universidad

SUMARIO: I. INTRODUCCION.-II. LA VIA DE ACCESO DE NAVARRA A LA AUTONOMIA PLENA A TRAVES DEL PROCEDIMIENTO PREVISTO EN LA DISPOSICION ADICIONAL PRIMERA DE LA CONSTITUCION ESPAÑOLA DE 1978.-III. EL SISTEMA DE DISTRIBUCION DE COMPETENCIAS EN LA LORAFNA: 1. TECHO COMPETENCIAL CON ARREGLO AL PRINCIPIO DE «UNIDAD CONSTITUCIONAL». 2. CONCRECIÓN DEL SISTEMA DE DISTRIBUCIÓN DE COMPETENCIAS: A) Competencias denominadas históricas. B) Competencias institucionales. C) Competencias extrainstitucionales. 3. TIPOLOGIA DE LAS FACULTADES Y COMPETENCIAS DE NAVARRA. 4. El CONCEPTO DE COMPETENCIAS EXCLUSIVAS. 5. LAS COMPETENCIAS DE DESARROLLO LEGISLATIVO Y EJECUCIÓN DE LA LEGISLACIÓN BÁSICA DEL ESTADO: Las competencias de desarrollo le-

(*) Texto de la conferencia pronunciada el día 4 de abril de 1984 en el Aula Magna de la Universidad de Navarra, en el marco del I Curso de Derecho Administrativo Foral de Navarra, organizado por el Departamento de Derecho Administrativo de la Universidad, y a cuyo titular, don Francisco González Navarro, agradecemos muy vivamente la autorización para publicarla en las páginas de esta Revista.

A la versión expuesta oralmente se le han añadido, para esta ocasión, las notas correspondientes. 
gislativo: sus diferencias con las competencias exclusivas. 6. LAS competencias de eJeCución: A) Primera consecuencia. B) Segunda consecuencia.-IV. CONSIDERACION FINAL.

\section{INTRODUCCION}

Al dar inicio a esta conferencia se hace preciso acotar el ámbito de la misma.

Sabido es que el título que aparece en el programa de este primer Curso de Derecho Administrativo Foral es el de "Competencias de Navarra». La extensión del mismo hace que nuestra disertación de hoy se circunscriba al análisis de las competencias de Navarra en la Ley Orgánica de Reintegración y Amejoramiento del Régimen Foral de Navarra (en adelante se citará abreviadamente LORAFNA), y en particular haré referencia a su sistema.

Ello nos llevará, si cabe, brevemente al análisis del procedimiento seguido por Navarra para acceder a su plena autonomía, así como el criterium de distribución de competencias, tal como se desprende de una atenta lectura de los artículos 2 y 3 y preceptos concordantes del Título II de la LORAFNA.

$\mathrm{Ni}$ que decir tiene, porque el empeño resultaría hic et nunc, que ocioso es proceder a realizar un análisis singular por materias o bloques de materias de las competencias de Navarra.

Dicho esto, podemos entrar en el primer aspecto de esta disertación.

\section{LA VIA DE ACCESO DE NAVARRA A LA AUTONOMIA PLENA A TRAVES DEL PROCEDIMIENTO PREVISTO EN LA DISPOSICION ADICIONAL PRIMERA DE LA CONSTITUCION ESPAÑOLA DE 1978}

Es conocido que Navarra accedió a su encaje constitucional en el Estado de las autonomías o Estado compuesto que delinea la Constitución de 1978 a través del vehículo singular de la LORAFNA (1). Este dato de "vía a la autonomía» es importante destacarlo

(1) Vid. nuestro trabajo «En torno a la Ley Orgánica de Reintegración y Amejoramiento del Régimen Foral de Navarra» y la bibliografía allí citada publicada en $R A P$, núm. 99. En relación con el instrumento (la LORAFNA) de acceso de Navarra a la autonomía plena, véase las consideraciones de S. MuÑoz Machado en su Derecho público de las Comunidades Autónomas, t. I, Madrid, 1982, págs. 276 y sigs. 
porque en nuestra Constitución, como veremos, vías de acceso y techos competenciales no son términos contrapuestos, sino absolutamente complementarios. En efecto, la llamada vía lenta de la autonomía, a través del artículo 143 de la Constitución Española, posibilita la asunción de las competencias contempladas en el artículo 148, más aquellas otras que ex artículo 150, apartados $1 .^{\circ}$ o $2 .^{\circ}$, puedan ser atribuidas, transferidas o delegadas a las Comunidades Autónomas.

Mientras que para las Nacionalidades o Regiones que hayan seguido la vía prevista en el artículo 151 y Disposición Transitoria 2. ${ }^{\mathrm{a}}$ su ámbito competencial se ve ampliado, ya que los Estatutos promulgados por esta vía pueden obtener, $y$ así ha sido en virtud de lo dispuesto en el artículo 148, 2, mayores competencias en el marco establecido en el artículo 149, 1, de nuestra Carta Magna.

¿Qué decir, en este punto, de la vía de Navarra a la autonomía plena, a través de la Disposición Adicional 1. ${ }^{a}$, a los efectos competenciales, que son los que aquí nos interesan? No voy a comentar aquí el procedimiento seguido por Navarra para acceder a su autonomía plena, porque ni es hoy tema que nos ocupa, y además ha sido ya abordado por otros conferenciantes, y no quisiera en este punto ser repetitivo.

Ahora bien, es necesario constatar que la vía de acceso de $\mathrm{Na}$ varra está justificada en función de los derechos históricos, y que los mismos determinan la vía de acceso y el techo competencial.

Que Navarra, la Comunidad Foral de Navarra, a través de la vía de acceso de la Disposición Adicional 1. a, se va a configurar con un techo competencial muy amplio nucleado en torno al concepto de unidad constitucional, que va a posibilitar un nivel competencial que podríamos denominar "propio» (2).

(2) Sobre el acceso de Navarra a la autonomía plena por la vía de la Disposición Adicional 1.", párrafo $10^{\circ}$, véanse las sentencias del Tribunal Constitucional 16/ 1984 , de 6 de febrero, y 28/1984, de 28 de febrero. En la primera de las referidas sentencias, el Tribunal Constitucional declara (fundamento jurídico $3 .^{\circ}$ ): «El hecho de que el acceso de Navarra a su actual régimen autonómico se haya llevado a cabo por una vía peculiar -mediante lo previsto en la Disposición Adicional 1. Constitución- y de que la denominación utilizada en la Ley Orgánica 13/1982, de 10 de agosto, de Reintegración y Amejoramiento del Régimen foral de Navarra, para referirse a la Entidad autónoma por ella regulada, sea la de Comunidad Foral y no la de Comunidad Autónoma, no supone que no le sean aplicables esas disposiciones constitucionales o que quede al margen de ellas».

En la segunda reconoce el sistema peculiar de acceso a la autonomía plena partiendo del Real Decreto de 26 de enero de 1979 de ordenación de las instituciones forales, y se declara (fundamento jurídico 2.\%): «La Ley Orgánica de Reintegración y Amejoramiento viene a reformar y modernizar el Régimen foral teniendo en cuenta 
Ello nos exige analizar el contenido y alcance de la LORAFNA y entrar, en consecuencia, a examinar el techo competencial con arreglo al principio de «unidad constitucional».

\section{EL SISTEMA DE DISTRIBUCION DE COMPETENCIAS EN LA LORAFNA}

1. TECHO COMPETENCIAL CON ARREglo AL PRINCIPIO DE «UNIDAD CONSTITUCIONAL»

A efectos de dar respuesta a las cuestiones planteadas, es necesario analizar el contenido y alcance de la LORAFNA (3). En este sentido, el artículo $2 .^{\circ}$ de la misma dispone:

«1. Los derechos originarios e históricos de la Comunidad Foral de Navarra serán respetados y amparados por los poderes públicos, con arreglo a la Ley de 25 de octubre de 1839 , a la Ley Paccionada de 16 de agosto de 1841 y disposiciones complementarias, a la presente Ley Orgánica y a la Constitución, de conformidad con lo previsto en el párrafo $10^{\circ}$ de su Disposición Adicional 1. ${ }^{\mathrm{a}}{ }$.

A continuación del número 1 del artículo $2 .^{\circ}$ transcrito, el número 2 del mismo precepto establece:

«2. Lo dispuesto en el apartado anterior no afectará a las instituciones, facultades y competencias del Estado inherentes a la unidad constitucional».

Congruentemente con la declaración formulada en el artículo $2 .^{\circ}$ de la LORAFNA, que es el marco que contiene la explicitación de los derechos originarios e históricos de la Comunidad Foral, el artículo 3.॰ de la Ley expresa:

«De acuerdo con la naturaleza del Régimen foral, su amejoramiento, en los términos de la presente Ley Orgánica, tiene por objeto:

lo dispuesto en la Disposición Adicional 1., siguiendo el proceso de reintegración y amejoramiento reflejado en el Decreto anterior.

(3) Sigo en este punto mi trabajo anteriormente citado. 
1. Integrar en el Régimen foral de Navarra todas aquellas facultades y competencias compatibles con la unidad constitucional.

3. Garantizar todas aquellas facultades y competencias propias del Régimen foral de Navarra".

De los preceptos anteriormente citados, interpretados armónicamente, se desprende:

1. Que la Comunidad Foral de Navarra tiene garantizadas, por determinación expresa del artículo $3 .^{\circ}, 3$, todas aquellas facultades y competencias "propias» del Régimen foral.

No voy a hacer una enumeración de cada uno de los sectores en los que Navarra ostenta competencias en virtud de su tradicional Régimen foral, cuestión que nos llevaría demasiado lejos y no es el objeto de la presente conferencia, pero sí cabe indicar que será un dato a considerar y, por tanto, a tener en cuenta a efectos de interpretación del desarrollo legislativo de la presente Ley.

2. Que se integran en el Régimen foral todas aquellas facultades y competencias compatibles con la unidad constitucional.

He aquí el concepto básico que constituye el punto de arranque del sistema competencial de la Comunidad Foral de Navarra.

"Integrar», dice el Diccionario de la Real Academia Española de la Lengua, significa: "Completar uno un todo con las partes que faltaban".

A estos efectos no es baladí afirmar que mientras todos los Estatutos promulgados hacen referencia a que con el Estatuto, que es su norma institucional básica, "se constituyen en Comunidad Autónoma", Navarra constituye ya una Comunidad Foral con régimen, autonomía e instituciones propias (art. 1. ${ }^{\circ}$ de la Ley).

Integrar en una todas aquellas facultades y competencias compatibles con la unidad constitucional.

La cuestión relativa al techo competencial hay que referirla al concepto, que aquí es nuclear, de «unidad constitucional».

El principio de unidad constitucional en el campo competencial se configura como un concepto jurídico indeterminado, que deberá ser interpretado en el sentido de que todo aquello que no sea esencial al Estado, es decir, que éste no haya reservado para sí en la nueva distribución del poder que opera la Constitución de 1978, puede ser integrado en el Régimen foral de Navarra. 
Este concepto jurídico indeterminado de «unidad constitucional» interpretado de una manera dinámica es el que ha posibilitado que la Comunidad Foral de Navarra haya alcanzado el techo competencial, que a continuación expondré.

Pero ¿qué es, a efectos competenciales, «unidad constitucional»? Un estudio exhaustivo del término y el significado del mismo nos podría llevar muy lejos, y no es mi propósito el aburrirles, pero sirvan a modo de esquema provisional las consideraciones siguientes:

Es sabido de todos ustedes que nuestra Constitución se fundamenta, a efectos de distribución territorial, en dos principios capitales: unidad de la Nación española (4) y el derecho a la autonomía de las Nacionalidades y Regiones que la integran, que en nuestro caso, en Navarra se ve completado con el principio del amparo $y$ respeto de los derechos históricos de los territorios forales. A efectos de traducir estos dos principios a ámbitos de poder, la Constitución Española reserva al Estado una serie de títulos competenciales para preservar lo que el constituyente entiende es expresión del principio de unidad, títulos que se contienen en el artículo 149 de la Constitución Española. A su vez, el constituyente quiso, al objeto de reconocer y garantizar el derecho a la autonomía de las Nacionalidades y Regiones, reservar un ámbito competencial "propio" para las Comunidades Autónomas, que se concreta fundamentalmente en el artículo 148 de la Constitución Española; sin embargo, debido al rígido sistema de dos listas que establece nuestra Constitución, ésta se vio obligada a atenuar los títulos conferidos al Estado con carácter exclusivo, permitiendo ya directamente - Comunidades constituidas por la vía rápida-, ya a través del transcurso de cinco años - Comunidades constituidas por la vía lenta-, que las Comunidades Autónomas pudieran ampliar su ámbito competencial en el marco del artículo 149. Para ello utilizó una técnica poco precisa, lo que le obligó a emplear expresiones tales como «legislación, normas básicas y bases», que fueron aprovechadas por las Comunidades que tramitaron sus primeros Estatutos de Autonomía, lo que, en consecuencia, moduló la afirmación del párrafo primero del artículo 149 de "que el Estado tiene competencia exclusiva

(4) Vid. S. Muñoz Machado: Derecho público de las Comunidades Autónomas, tomo I, Madrid, 1981, págs. 199 y sigs. Véase, asimismo, su artículo «Los principios constitucionales de unidad y autonomía y el problema de la nueva planta de las Administraciones públicas», en $R A P$, núm. 102, vol. II. 
sobre las siguientes materias". Ello posibilitó la concreción de un generoso techo competencial para este tipo de Comunidades. Afirmación ésta, la del artículo 149 de la Constitución Española, que debía verse también modulada por la Disposición Adicional 1. ${ }^{2}$, que, como es sabido, sanciona el principio de amparo y respeto de los derechos forales de los territorios históricos.

Pues bien, todo ello me lleva a concluir que en materia competencial «unidad constitucional» es el contenido del artículo 149, modulado por los ámbitos competenciales asumidos por los Estatutos y por el principio sancionado en la Disposición Adicional $1 .^{\mathrm{a}}$ Principio éste que encuentra su sanción con la interpretación del Tribunal Constitucional, intérprete supremo de nuestra Carta Magna.

Esta afirmación explica, como diré más adelante, que además de asumir las competencias a que se refiere el artículo 148, Navarra entre en el ámbito del artículo 149 integrando todas aquellas competencias que las Comunidades Autónomas, constituidas al amparo del artículo 151, han reservado como propias.

De una manera gráfica y a modo de resumen, se puede concluir que la Comunidad Foral de Navarra ha visto garantizadas por la LORAFNA sus competencias históricas; integradas en su Régimen foral las competencias que ex artículos 148 y 149 han asumido las Comunidades Autónomas constituidas por la vía del artículo 151 de la Constitución; posibilitadas, por efecto de una interpretación dinámica del concepto de unidad constitucional, la atribución, delegación o transferencia de todas las facultades y competencias que la legislación del Estado atribuye, delegue o transfiera a las Comunidades Autónomas o Provincias. Dicho en otros términos, competencias que podríamos denominar históricas (art. $33^{\circ}, 1$, en relación con el art. 39, 1, a) ), institucionales (art. $3 .^{\circ}$, 1 , en relación con el artículo $39,1, b$ ) ) y las extrainstitucionales (art. $3 .^{\circ}, 1$, en relación con el artículo $39,1, c)$, y 39, 2).

Lo hasta aquí expuesto debe entenderse sin perjuicio de lo dispuesto en la Disposición Adicional 1. ${ }^{a}$ de la LORAFNA.

«La aceptación del régimen establecido en la presente Ley Orgánica no implica renuncia a cualesquiera otros derechos originados e históricos que pudieran corresponder a Navarra, cuya incorporación al ordenamiento jurídico se llevará a cabo, en su caso, conforme a lo establecido en el artículo 71», es decir, mediante pacto.

En respuesta a la pregunta que antes nos hicimos, cabe indicar que el techo competencial de la Comunidad Foral de Navarra no es 
similar al nivel de competencias correspondiente a una Comunidad Autónoma constituida al amparo de lo dispuesto en el artículo 143, ya que le supera, ni se identifica plenamente con el criterium competencial correspondiente a un Estatuto tramitado por la vía del artículo 151 de la Constitución, si bien se le aproxima, y ello en función de la vía de los derechos históricos, que en esta vía también singularizan a Navarra.

\section{CONCRECIÓN DEL SISTEMA DE DISTRIBUCIÓN DE COMPETENCIAS}

Examinado el techo competencial de la Comunidad Foral de Navarra es necesario indagar cuál es su sistema de competencias, o dicho en términos más estatutarios, cuál es su sistema de distribución de competencias.

En este punto, y como cuestión previa, es necesario indicar que la LORAFNA no se ha despegado del sistema de distribución de competencias que siguieron los Estatutos de Autonomía. (Aquí hay que abrir un paréntesis y aseverar que, a excepción de pequeños detalles del sistema de distribución de competencias inserto en los diferentes Estatutos de Autonomía, cualquiera que sea su techo competencial siguen los modelos vasco y catalán.) Ello, sin embargo, no nos puede llevar a asimilaciones fáciles en cuanto a su naturaleza con los Estatutos de Autonomía, por lo que expondré a continuación.

Para mí es diáfano, como he tratado de exponer, que el concepto de unidad constitucional se traduce, a efectos de delimitar lo básico, al artículo 149 de la Constitución, con las modulaciones indicadas; pues bien, si ello es así, parece obvio que a efectos de acomodarse a la técnica utilizada por la Constitución, la LORAFNA utilice un sistema de distribución de competencias análogo al de los Estatutos de Autonomía.

Es conocido que la Constitución Española sigue, como ya he indicado incidentalmente más arriba, a diferencia de precedentes históricos, como la Constitución de 1931 o modelos de Derecho comparado, el sistema de dos listas, las que se contemplan en los artículos 148 y 149 de la Constitución Española; pues bien, este sistema de dos listas ha determinado, sin duda, el sistema de distribución de competencias de los Estatutos de Autonomía, y en nuestro caso de la LORAFNA, pero amén de hablar de técnicas es necesario que vayamos ya perfilando el sistema. A mi juicio, en atención a lo 
que he dicho anteriormente, y de acuerdo a lo que sancionan los artículos 2 y 3 de la LORAFNA, el sistema de competencias de Navarra queda de la siguiente manera:

\section{A) Competencias denominadas históricas (5)}

Hacen referencia a todas aquellas facultades y competencias que Navarra, a través de sus órganos, ejerce al amparo de lo establecido en la Ley Paccionada de 1841 y disposiciones complementarias (artículo 39, 1, a) ).

\section{B) Competencias institucionales}

Que son todas aquellas facultades y competencias que expresamente se le integran por la presente Ley.

Cabe diferenciar en este segundo tipo de competencias entre competencias exclusivas de Navarra, que, a su vez, pueden distinguirse entre competencias que se dicen exclusivas en virtud de lo dispuesto en los artículos 44, 48 y 56, y competencias que son exclusivas en virtud de su Régimen foral (así, arts. 45, 46, 49 y 50), competencias de desarrollo legislativo y ejecución de la legislación básica del Estado (arts. 50, 2; 53; 54; 55, y 57) y competencias de ejecución de la legislación del Estado (art. 58).

\section{C) Competencias extrainstitucionales}

Son aquellas que pueden ser contempladas desde una doble vertiente, es decir, ya sean las competencias que la legislación del Estado atribuye, transfiera o delegue con carácter general a las Comunida. des Autónomas o Provincias, ya sean todas aquellas facultades y competencias que no estando comprendidas en las anteriores, a iniciativa de la Diputación, sean transferidas, atribuidas o delegadas a Navarra.

(5) Sobre las competencias que denomino históricas, véase la interesante doctrina contenida en la sentencia del Tribunal Constitucional 11/1984, de 2 de febrero (conflicto de competencia sobre autorización para emitir deuda pública a la Comunidad Autónoma del País Vasco), que declara «que las fuentes de las que nacen las competencias de los territorios históricos, por un lado, y de las Comunidades Autónomas, por otro, son necesariamente distintas. Los territorios históricos son titulares de 'derechos históricos', respetados, amparados y sujetos a la actualización en el marco de la Constitución Española y de los Estatutos de Autonomía, en virtud de lo dispuesto en la Disposición Adicional 1." de la Constitución, por lo que la delimitación de las competencias de tales territorios podría exigir una investigación histórica acerca de cuáles sean tales 'derechos's. 
Dado lo extenso que sería el examinar este sistema de competencias tal como se han clasificado, conviene centrar nuestro análisis en el día de hoy a las denominadas competencias institucionales, y ello por varias razones. La primera, y esto hace relación al conjunto de competencias denominadas históricas, las mismas requieren tanto su propia definición para poder ser ejercitadas como para ampliar, como veremos más adelante, el tipo de competencias que denomino institucionales y que se rotulan exclusivas, de un estudio que delimite cuáles son por sectores o materias las competencias que Navarra ejerce en virtud de sus títulos históricos, en este caso, Ley Paccionada y disposiciones complementarias.

Como se comprenderá fácilmente, no es éste el momento ni el lugar para proceder a tan sugestiva tarea, como impropia finalidad de esta conferencia.

Asimismo, existe otra categoría de competencias que he denominado extrainstitucionales que, a pesar de ser previsión legal, sin embargo sí requieren, a mi juicio, un breve comentario antes de entrar de lleno en el examen de las denominadas competencias institucionales.

Las que he denominado competencias extrainstitucionales están contempladas en el artículo $39,1, c)$, y en el apartado 2 del referido precepto. Conviene distinguirlos.

De la lectura de la letra a) del apartado $10^{\circ}$ del artículo 39 se deduce que corresponden, es decir, pertenecen a Navarra. Sin embargo, la eficacia de esas competencias que ya corresponden se condiciona a un acto volitivo del Estado, a que por la legislación del mismo se atribuya, transfiera o delegue con carácter general a las Comunidades Autónomas o a las Provincias.

Ahora bien, ¿cuáles son los títulos constitucionales que habilitan al Estado para atribuir, transferir o delegar facultades y competencias que son exclusivas del Estado, porque si no lo fueran hubieran sido ya integradas o incorporadas por la propia LORAFNA?

Sin duda, hay que afirmar que en el artículo 150, apartados 1 y 2 , de la Constitución Española, que disponen, el primero, que las Cortes Generales podrán atribuir a todas o a alguna de las Comunidades Autónomas la facultad de dictar por sí mismas normas legislativas en el marco de los principios, bases y directrices fijados por una ley estatal, posibilitando la asistencia de controles parlamentarios al margen de los judiciales. 
El segundo (6), que «el Estado podrá transferir o delegar en las Comunidades Autónomas, mediante ley orgánica, facultades correspondientes a materia de titularidad estatal que por su propia naturaleza sean susceptibles de transferencia o delegación.

Qué lectura armónica puede hacerse de los preceptos transcritos:

1. Que la previsión a que se refiere la letra a) del apartado $1 .^{\circ}$ parece querer acomodarse a los supuestos que prevé el artículo 150, 1 y 2, de la Constitución.

2. Que la referencia a atribución, transferencia o delegación de facultades y competencias ha de ser con carácter general, y este dato conviene subrayarlo porque las Cortes Generales pueden atribuir facultades y competencias singularizadamente, casos, por ejemplo, de las Leyes Orgánicas 11 y 12/1982, de 10 de agosto, de transferencia a las Comunidades Canaria y Valenciana de competencias en materia de titularidad estatal o bien a iniciativa de la Diputación singularizadamente.

3. Que los referidos apartados del artículo 39 tratan de prever agotadoramente todas las posibles transferencias que el Estado realice a las Comunidades Autónomas, sea con carácter general o bien singularizadamente.

\section{TiPología dE LAS FACULTADES y COMPETENCIAS DE NAVARRA}

Entramos en este apartado, tal como había indicado anteriormente, en el análisis de las competencias que denomino institucionales, es decir, aquellas competencias que se integran en el Régimen foral, ya que son compatibles con la unidad constitucional.

Estas competencias institucionales se regulan en el capítulo segundo - -Delimitación de facultades y competencias»- del Título II de la LORAFNA - "Facultades y competencias de Navarra», artículos 44-58-, y las mismas pueden clasificarse, a su vez, en tres tipos: 1. Aquellas cuya titularidad corresponde a Navarra. 2. AqueIlas otras por las que corresponde a Navarra el desarrollo legislativo y ejecución de la legislación básica del Estado. 3. Por último, las denominadas competencias de ejecución.

Cabe aquí indicar que a cada una de estas calidades competen-

(6) Vid., sobre el artículo 150, 2, de la Constitución Española, el artículo de M. Bassols Coma y J. M. Serrano Alberca: «El artículo 149 de la Constitución en relación con el artículo 150, 2: análisis de la delegación en materia de las competen. cias estatales excluidas», en $R A P$, núm. 97. 
ciales le corresponden unas potestades determinadas. Así, en relación con las competencias cuya titularidad exclusiva corresponde a Navarra, la Comunidad Foral dispone de las potestades legislativa, reglamentaria y de administración (art. 40,1). En aquellas materias en las que Navarra ostenta el desarrollo legislativo de la legislación básica del Estado se ordenan potestades de desarrollo legislativo, reglamentario y de administración (art. 41, 1).

Finalmente, a las competencias de ejecución le corresponden la potestad reglamentaria para la organización de sus propios servicios de administración y revisora en vía administrativa (art. 42, 1).

De esta suerte de potestades que corresponden a la tipología competencial antes enunciada, cabe observar que supone un intento de clarificación respecto a lo dispuesto en algunos Estatutos de Autonomía tramitados ex artículo 151 de la Constitución.

Voy a referir a continuación dos importantes problemas que han sido, en parte, abordados por la doctrina y por el Tribunal Constitucional:

1. La noción de competencias exclusivas (7).

2. Las competencias de desarrollo legislativo, su diferenciación de las competencias exclusivas.

\section{EL CONCEPTO DE COMPETENCIAS EXCLUSIVAS}

La LORAFNA, como ya se ha indicado, ha incorporado a la misma la técnica de atribución de competencias exclusivas ejercidas por los Estatutos de autonomía especiales, consistente en asumir como competencias de titularidad exclusiva aquellas competencias que el artículo 148 de la Constitución posibilita, ampliadas dentro del marco establecido en el artículo 149 de la misma.

A esta técnica de asunción de competencias a las que se adjetiva de exclusivas y que han sido tildadas de «equívocas» por el Tribunal

(7) Sobre la noción de competencias exclusivas, vid. especialmente S. MuÑoz MACHADO: «La interpretación estatutaria del sistema constitucional de distribución de competencias», en $R$. D. Pol., núm. 5 (UNED), págs. 61 y sigs., y también su Derecho público, t. I, págs. 357 y sigs.; J. Salas: «Los poderes normativos de la Generalitat de Catalunya», en $R E V L$, núm. 205, págs. 16 y sigs.; García De EnTERrf́a: «La significación de las competencias exclusivas del Estado en el sistema autonómico», en $R E D C$, núm. 5 , págs. 71 y sigs., y la primacía del título VIII de la Constitución, "Introducción al estudio del artículo 149 de la Constitución», en REDA, núm. 33, páginas 277 y sigs.; A. BLASCO: «Sobre el concepto de competencias exclusivas», en REDA, núm. 23, págs. 314 y sigs.; LASAgABASTER HerRARTE: La potestad legislativa de las Comunidades Autónomas, Oñati, 1982, págs. 177 y sigs. 
Constitucional (sentencias del Tribunal Constitucional de 16 de noviembre de 1981 y 8 de febrero de 1982), hay que añadir el plus de que en la LORAFNA se contemplan competencias de titularidad exclusiva de doble vertiente. Las que refuerzan el carácter de competencias exclusivas en función de la virtualidad del Régimen foral de Navarra (arts. 49 y 50) y aquellas otras que no lo invocan (artículos 44,48 y 56). Ello nos obliga a examinar, en primer lugar, el concepto de competencia exclusiva. Dicho concepto ha sido ya estudiado por diversos autores: GaRcía de ENTERRÍA (8), MUÑoz MACHADO (9) y Salas (10), al hilo de la interpretación del régimen de distribución de competencias de los primeros Estatutos de autonomía, y han cuestionado de una manera u otra el carácter de competencia exclusiva como sinónimo de plena disponibilidad de una determinada materia, y han tratado de reconducirla hacia lo que la doctrina denomina competencias compartidas. $\mathrm{Y}$ así, por ejemplo, el profesor SALAS indica que si no se confunden competencias separadas y competencias exclusivas, es posible desde una perspectiva procedimentalizada, es decir, por fases de un procedimiento simple o por procedimientos de uno complejo, propugnar la atribución de competencias exclusivas a favor de las Comunidades Autónomas, concluyendo que el concepto de competencia exclusiva no es incompatible con el de compartida o concurrente.

El profesor LASAGABASTER (11) ha perfilado una definición de competencia exclusiva, muy al hilo de los Estatutos Vasco y Catalán, en el sentido de que por competencias legislativas exclusivas debe entenderse aquella que permite la utilización de la potestad legislativa sobre una materia, sobre una parte de una materia o sobre una fase procedimental, sin que en la misma pueda participar ningún otro Ente y siempre que como tal competencia exclusiva esté denominada en los Estatutos.

Sin negar lo posibilista de la definición a efectos de "explicitar» el régimen de las potestades legislativas, entiendo que en la LORAFNA, como a continuación trataré de explicar, "competencias exclusivas» no pueden ser entendidas, en su generalidad, como competencias separadas, sino que es necesario adoptar una perspectiva singularizada; entiendo que la titularidad exclusiva puede referirse a una parcela o sector de la materia, distribución horizontal, o bien

(8) Vid. Garcf́a de Enterrfa, op. cit., en especial «La significación», pág. 71.

(9) Vid. Muñoz Machado: Derecho público, t. I, pág. 317.

(10) Vid. Salas: "Los poderes», pág. 205.

(11) Vid. Lasagabaster Herrarte: "La potestad», págs. 117 y sigs. 
a una fase o momento de un procedimiento simple o a un procedimiento específico dentro de un complejo procedimental de actualización de potestades públicas sobre aquéllos, situándolos en la esfera de las competencias compartidas, y entiendo asimismo que este esquema es válido tanto para los preceptos que atribuyen competencias exclusivas invocando la virtualidad del Régimen foral, como para aquellos otros que no lo invocan, encontrando su explicación en que ambas clases de preceptos salvan la competencia del Estado bajo las fórmulas de: «sin perjuicio de las facultades», «conforme a la legislación general», "de acuerdo con los principios básicos», etc.

Lo que hasta aquí se ha indicado es importante, ya que supone admitir que no en todos los supuestos que contemplan los artículos 44, 48, 49, 50 y 56 estamos en presencia de competencias de titularidad exclusiva, y esta circunstancia incide en tres órdenes de supuestos:

En primer lugar, porque el artículo 40, 3, dispone que el Derecho navarro en las materias de competencias exclusivas será aplicable con preferencia a cualquier otro. Si no todas las competencias son conceptualmente exclusivas, la regla del referido precepto se verá sustituida por la cláusula del artículo 149, 3.

En segundo lugar, porque la competencia para conocer de los actos dictados por la Administración Foral corresponderá en el orden contencioso-administrativo, en todas las instancias y grados, a los órganos jurisdiccionales radicados en Navarra.

Si bien cabe indicar que el precepto comentado no se refiere expresamente a los actos dictados en el ejercicio de competencias exclusivas, es sostenible que se refieran a ella, ya que es función de esa exclusividad por la que se agotan todas las instancias y grados en los órganos jurisdiccionales radicados en Navarra, aunque habrá que estar, en todo caso, a lo que la futura Ley Orgánica del Poder Judicial disponga.

Finalmente, incidirá en el régimen de convenios para la gestión y prestación de servicios propios correspondientes a materia de competencia exclusiva de la Comunidad Foral, y ello porque el régimen de aprobación de aquéllos es distinto del de los llamados acuerdos de cooperación.

A modo de resumen del régimen de las competencias exclusivas en la LORAFNA puede indicarse:

1. De ciertas materias incluidas en los artículos 44 (apartados 
$2,3,5,10,11,12,13,14,15,16,17,18,19,20,21,22$ ), 48, 49 (apartados $1-a, 1-c, 1-f, 1-g, 1-h$ ) y 50 (apartados $1-b, 1-c$ ) puede predicarse que estamos en presencia de competencias exclusivas, entendidas éstas como separadas.

2. De las competencias no enumeradas explícitamente en el apartado anterior, puede aseverarse que no estamos en presencia de competencias separadas, sino de competencias compartidas, así como en los supuestos de los artículos 46, 49 y 50, donde bajo el rótulo de exclusivo se salva la competencia del Estado.

\section{LAS COMPETENCIAS DE DESARROLLO LEGISLATIVO Y EJECUCIÓN DE LA LEGISLACIÓN BÁSICA DEL ESTADO}

Las competencias de desarrollo legislativo: sus diferencias con las competencias exclusivas

La LORAFNA dedica los artículos 50,$2 ; 53 ; 54 ; 55$, y 57, a las llamadas competencias de desarrollo legislativas y ejecución de la legislación básica del Estado. Como ya se ha expuesto, a tal calidad competencial le corresponden una serie de potestades que ya han sido referidas (art. 41,1 ).

Dichas competencias de calidad, sin duda alguna normativa, se caracterizan porque la legislación de detalle debe ejercitarse de conformidad con normas básicas que dicte el Estado.

Si examinamos brevemente la materia a que estas competencias se refieren, observamos que hacen relación a:

- Montes de propiedad de los particulares.

- Sanidad interior, higiene y Seguridad Social.

- Radiodifusión, televisión y prensa.

- Sistema de responsabilidad de las Administraciones públicas.

- Expropiación forzosa.

- Medio ambiente.

- Ordenación del crédito, Banca y seguros.

- Régimen minero y energético.

La problemática jurídica de más enjundia que se plantea en torno a las competencias de desarrollo legislativo es la siguiente: ¿Qué diferencia cualitativa existe entre las competencias exclusivas que enumera la LORAFNA, pero que salvan la competencia estatal 
a través de fórmulas como: «sin perjuicio de», "de acuerdo con», y las competencias propiamente establecidas como de desarrollo legislativo?

El examen de los artículos 40, 1 y 2, y 41, 1 y 2, nos induce, prima facie, a considerar que las diferencias cualitativas no pueden ser muy ostensibles.

Veámoslo: el artículo 40, apartados 1 y 2, dispone que en el supuesto de competencias exclusivas de Navarra, las potestades que corresponden a las mismas deben ejercitarse en los términos previstos en la presente Ley y en la legislación del Estado a la que la misma hace referencia.

A su vez, el artículo 41, apartados 1 y 2, establece que en las materias en las que a Navarra corresponde el desarrollo legislativo y ejecución de la legislación básica del Estado, las potestades de desarrollo legislativo, es decir, las normativas, deberán ejercitarse de conformidad con las normas básicas que dicte el Estado.

$¿$ Es posible distinguir entre las locuciones «legislación del Estado a la que la misma hace referencia» $\mathrm{y}$ «normas básicas que dicte el Estado»?

Formulada la pregunta en otros términos, $¿$ son intercambiables las locuciones legislación del Estado, legislación básica y normas básicas que dicte el mismo?

Las locuciones legislación, legislación básica, bases, aparecen recogidas en los distintos apartados del artículo 149 de la Constitución.

La doctrina, en concreto los profesores SalaS y MuÑoz MACHaDo (12), se ha pronunciado con matices diferentes acerca de la inintercambiabilidad de los términos o locuciones expresados, así como con matices nuestro más Alto Tribunal en materias constitucionales.

Así, el Tribunal Constitucional ha perfilado al hilo de sus sentencias de fecha 5 de noviembre de 1981,4 de mayo y 14 y 30 de junio de 1982, el concepto de legislación en el sentido de que el mismo debe entenderse en forma material y que no puede asimilarse sin más al conjunto de normas escritas, sino que el concepto o locución de «legislación», manejado por el artículo 149 de la Cons-

(12) Vid. los trabajos del Profesor SaLAs: «El desarrollo estatutario de la jurisprudencia del Tribunal Constitucional», publicado en $R V A P$, núm. 5, y «Estatutos de Autonomía, Leyes Básicas y Leyes de Armonización", en RAP, núms. 100-102, donde se pronuncia acerca de las locuciones «legislación básica», "normas básicas» y «bases», como términos intercambiables con un contenido distinto; y del Profesor Mữoz MaCHADo: Derecho público, págs. 417 y sigs. 
titución Española, comprende tanto leyes formales como reglamentos ejecutivos de las mismas.

También ha tenido ocasión el Tribunal Constitucional de pronunciarse acerca de las locuciones legislación básica y bases, si bien, en relación con la primera, no ha llegado a una delimitación tan nítida como con el concepto de «legislación». Sin embargo, en relación con «bases", el Tribunal Constitucional ha manejado los siguientes criterios:

1. Que el concepto de bases o legislación básica no es un concepto formal, sino material (sentencia de 28 de julio de 1981). «El ejercicio de la competencia estatal para el establecimiento de las bases o de la legislación básica no requiere en modo alguno la promulgación de leyes de bases o de leyes marco».

2. Que puesto que es una noción material, a la definición de lo básico pueden contribuir no sólo las leyes, sino también las normas reglamentarias, así ha dicho el Tribunal Constitucional (sentencia de 28 de julio de 1982). "De esta noción material de bases se infiere que unas normas no son básicas por el mero hecho de estar contenidas en una ley y ser en ella calificadas como tales».

Doctrina ésta que debe entenderse corregida por las sentencias de 28 de abril y 20 de mayo de 1983, ya que la primera de ellas apunta «que lo que no podrá hacer el Gobierno es definir en términos generales y por Real Decreto lo que es básico, pues es al legislador constitucional a quien corresponde establecer lo que entiende por bases de una materia. La segunda sentencia no excluye que el aspecto nuclear de lo básico pueda ser completado mediante normas reglamentarias en aquellos supuestos en que el desarrollo normativo con eficacia en toda la Nación venga exigido por la vigencia efectiva de las bases establecida por la ley.

Un tercer criterio consiste en admitir que las competencias sobre las bases incluye también las competencias de simple ejecución. El Tribunal lo ha entendido así: 1) cuando el acto de ejecución afecta directamente a intereses de diversas Comunidades Autónomas; 2) o la ejecución comporta por necesidades intrínsecas de la materia básica en cuestión márgenes tan amplios de discrecionalidad que sólo puede ser encomendada a instancias que no tienen a su cuidado intereses peculiares distintos de los puramente generales; 3) o bien cuando una situación de urgencia obliga al poder público que ha tenido conocimiento de la misma a actuar directamente para evitar 
daños irreparables, o todo aquello que sea preciso para la preservación de la normativa de una institución considerada como básica debe ser entendido, asimismo, como idóneo por vía de consecuencia o de conexión (13).

Pues bien, contestando a la pregunta que me había formulado, ya sea considerando que los conceptos no son intercambiables, por cuanto la locución "legislación" es equivalente a ley y reglamento, o las expresiones legislación básica o bases contemplen calidades competenciales legislativas, reglamentarias y de ejecución, con las matizaciones que hemos puesto de manifiesto, lo cierto es que, a mi juicio, la respuesta no puede ser enteramente positiva, ya que si examinamos la legislación del Estado, a la que las llamadas competencias exclusivas hacen referencia, observamos que se refieren asimismo a la legislación básica del Estado (art. 44, 6), de acuerdo con los principios básicos (art. 44, 24), etc., si bien notamos también que en ocasiones las referencias son a la legislación general del Estado (art. 44, núms. 23 y 26), o como en el número 28 del artículo 44, a la legislación mercantil. Se puede, pues, afirmar que las diferencias cualitativas entre las leyes que emanen del Parlamento de Navarra en ejercicio de un tipo $u$ otro de competencias no van a ser cualitativamente distintas.

A ello es necesario añadir la siguiente consideración. La primera es que, y a los efectos de la cuestión planteada, que las locuciones que aparecen en los artículos 40,2, y 41, 2, deben reconducirse a la cuestión anteriormente apuntada de la compartición de la materia, ya que desde la calificación objetiva como competencia compartida podrá, en supuestos singulares, $\mathrm{y}$ en base al deslinde competencial realizado por el Tribunal Constitucional, señalar qué diferencias existen, pero sólo desde la perspectiva de la compartimentación, ya que, como ha señalado el Tribunal Constitucional, en su sentencia de 8 de febrero de 1982, "resulta aventurado cualquier razonamiento que intente arrancar de la diferencia existente entre una competencia atribuida con carácter exclusivo, sin perjuicio de la legislación básica, que corresponde al Estado, y una competencia para legislar y ejecutar que debe hacerse en el marco de la legislación básica del Estado», podrá motivarse la "mejor» o "más estrecha sujeción» a la «legislación del Estado» en un supuesto o en otro.

(13) Vid. el voto particular formulado por el Magistrado constitucional don Francisco Rubio Llorente a la sentencia del Tribunal Constitucional 25/1983, de 7 de abril. 


\section{LAS COMPETENCIAS DE EJECUCIÓN}

En cuanto a las competencias de ejecución (14), es necesario proceder a su clasificación de acuerdo con el esquema del sistema de competencias que hemos elaborado más arriba.

Hemos indicado que, en el marco de lo que he denominado competencias institucionales, existen competencias exclusivas, entendidas éstas como competencias separadas y competencias compartidas.

Veíamos, además, que ciertas competencias exclusivas debían ser entendidas como competencias compartidas; a ello hay que añadir las competencias de ejecución strictu sensu de la legislación del Estado.

Veamos, pues, qué competencias de ejecución y cuál es su calidad o contenido.

En primer lugar, es necesario señalar que en el ámbito de las competencias que hemos denominado exclusivas, entendidas éstas como separadas o compartidas, aunque se puede predicar de ellas la exclusividad respecto a un sector de materia o una fase de un procedimiento, hay que indicar que con arreglo al principio de la plena disponibilidad sobre la misma, así como por la propia doctrina de la "naturaleza de las cosas", corresponde a Navarra ejecutar su legislación exclusiva y, por ende, y con mayores títulos, sus competencias históricas. Aquí habría que incluir todas las competencias que hemos tildado de exclusivas.

En segundo lugar, hay que hacer mención a la denominada legislación de desarrollo y ejecución de la legislación, que se refiere a los artículos 53, 54, 55, 56 y 57 de la LORAFNA.

Aquí es necesario deslindar lo que es legislación de desarrollo, de lo que es ejecución de la legislación o normas básicas del Estado.

En relación con el desarrollo hemos dicho antes que son competencias de naturaleza compartida, pero que determinan que su ámbito competencial de desarrollo legislativo es legislación propia de la Comunidad Foral y, por tanto, corresponde también a la Comunidad la ejecución de la misma.

Cuestión distinta es la ejecución de la legislación básica del Estado, porque puede corresponder al Estado la competencia para legislar y reglamentar una determinada materia, pero su ejecución corresponde a la Comunidad Foral.

(14) Vid. García dE EnTERrfa: La ejecución autonómica de la legislación del Estado, Ed. Civitas, Madrid, 1983; S. MuÑoz MACHADO: Derecho público, t. I, páginas 440 y sigs. 
Y, por último, nos encontramos con el supuesto previsto en el artículo 58 de la LORAFNA, en el que Navarra ostenta las facultades de ejecución de la legislación del Estado.

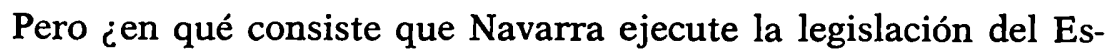
tado en las materias a que se refiere el artículo 58? Nos lo dice el artículo 42. En primer lugar, la potestad reglamentaria, pero reglamentaria interna, lo precisa el apartado a) «para la organización de sus propios servicios»; además, la potestad administrativa y la posibilidad de revisión en vía administrativa, pero todo ello de conformidad con las disposiciones de carácter general que en desarrollo de su legislación dicte el Estado.

Ahora bien, el contenido de dicho apartado, que acabo de enunciar, debe circunscribirse única y exclusivamente para la ejecución de la legislación del Estado, que es el supuesto a que se refiere el artículo 58, por cuanto entiendo que aquí es aplicable la doctrina del federalismo de ejecución, las técnicas de vigilancia y supervisión que la misma comporta.

No obstante lo expuesto, es necesario tener cuidado con las interpretaciones extensivas de esta doctrina y de las técnicas de vigilancia y supervisión que la misma comporta, pues lo que es lógico y se aplica sin traumas en países de estructura federal consolidada, puede no serlo tanto en nuestro país, donde, en primer lugar, la Constitución prevé que la ejecución de la legislación del Estado, en ciertas materias, se realice por las distintas Comunidades Autónomas, y en segundo lugar, y dicho sea sin aristas, cierto lastre centralista siguen rezumando los órganos centrales del Estado persona.

Como comprenderán ustedes, el cúmulo de interrogantes, de dudas, se apiñan en este punto.

Sin embargo, no podemos entrar en ellos; pero no quiero terminar mi intervención sin analizar, desde la perspectiva de las técnicas de ejecución, aunque fuera también brevemente, el significado del término "alta inspección».

Es sabido que el término nos es familiar aquí, en Navarra, por cuanto, entre otros textos, en el Convenio Económico de 1969, todavía hoy vigente, se emplea la fórmula.

Asimismo, no es necesario bucear mucho en la LORAFNA para encontrarnos con la expresión "alta inspección», artículos 47; 53, 3; $54,3 ; 58,1, b)$, etc.

¿Qué significa o qué es la alta inspección? En primer lugar, parece que estamos en presencia de un nuevo título que tiene el Estado, 
título que obedece a la traducción concreta del principio de unidad o interés general, pero es un título distinto de los títulos competenciales hasta ahora examinados. Nuestro Tribunal Constitucional ha tenido ya ocasión de pronunciarse, y así, en las sentencias del mismo de 22 de febrero de 1982 y 28 de abril de 1983.

En la primera de las sentencias citadas, nuestro más Alto Tribunal en cuestiones constitucionales conoció de un conflicto de competencias sobre funcionamiento en el País Vasco y Cataluña de la alta inspección del Estado en materia no universitaria. Varios son los aspectos que allí se abordaron, todos de un gran interés, pero nuestra atención se va a centrar en dos.

El primero hace referencia a la naturaleza del título "alta inspección»; así, nuestro Tribunal Constitucional dirá que «respecto a una materia concreta como es la enseñanza, la alta inspección puede ser considerada como un procedimiento licito de control que tiene un carácter esencialmente jurídico. La alta inspección garantiza exclusivamente el cumplimiento y garantía de aquellas facultades atribuidas al Estado".

$\mathrm{Y}$ el segundo al contenido. La alta inspección, dice el fundamento $3 .^{\circ}$ de la sentencia, aparece así revestida de un carácter jurídico no sólo en lo que concierne a un ejercicio, sino en cuanto a su contenido, pues recae sobre la correcta interpretación de las normas estatales, así como las que emanan de las asambleas comunitarias y cuando detectase fallos en el armónico desarrollo de las respectivas competencias propiciará su corrección.

La segunda sentencia se refiere también a un conflicto de competencia planteado por el Gobierno Vasco relativo a la materia sanitaria, en concreto, contra dos decretos sobre registro sanitario de alimentos y otro sobre coordinación y planificación sanitaria. En esta segunda sentencia, partiendo de la doctrina anteriormente anunciada, el Tribunal da un paso más y dispone que la «alta inspección» constituye una competencia estatal de vigilancia, pero no un control genérico e indeterminado que implique dependencia jerárquica de las Comunidades Autónomas respecto a la Administración del Estado, sino un instrumento de verificación que puede llevar, en su caso, la actuación de los controles constitucionales establecidos en relación con las Comunidades Autónomas, pero no a sustituirlos, convirtiendo a dicha alta inspección en un nuevo y autónomo mecanismo directo de control.

De lo expuesto pueden extraerse las siguientes consecuencias, no 
sin antes hacer la salvedad que no es posible incluir todas las referencias que en la LORAFNA se hace a la «alta inspección» en el mismo saco; me explico, lo que quiero hacer notar es que no tienen la misma calidad competencial, las competencias de ejecución que se contemplan en los artículos $47,53,54$ y 55, relativos a las competencias de la alta inspección de desarrollo legislativo, que las competencias de ejecución de la legislación básica, a que hacen también referencia los preceptos anteriormente citados, excluido el 47 , y la prevista en el artículo $18,1, b$ ).

\section{A) Primera consecuencia}

En el título relativo a la «alta inspección» que aparece en la LORAFNA, se hace referencia a una competencia estatal de vigilancia, que no de control strictu sensu, y, en consecuencia, dicha competencia de vigilancia puede dar lugar a la actuación, en su caso, de los controles previstos en la Constitución y en la LORAFNA, pero nunca strictu sensu a configurarse como título de control genérico.

\section{B) Segunda consecuencia}

El título relativo a la "alta inspección», a su contenido, en cuanto se configura fundamentalmente como de marcado carácter jurídico, en cuanto a la correcta interpretación desde un punto de vista estatal sobre actuaciones de contenido ejecutivo.

\section{CONSIDERACION FINAL}

No quiero terminar esta conferencia sin poner de manifiesto ante ustedes lo siguiente: La LORAFNA configura a la Comunidad Foral con un alto techo competencial, lo que comporta mayores posibilidades de autogobierno; sin embargo, esta profundización en la autonomía y, por tanto, en la mejora competencial de nuestro Régimen foral, supone un importante reto, el de transformar y acomodar una Administración orientada hacia la esfera local, en una Administración al servicio de una Comunidad Foral. De la audacia y oportunidad en las decisiones que adopten los órganos políticos, así como de la competencia y puesta al día de los funcionarios a su servicio, depende que dicho reto sea felizmente superado. 